\title{
Evaluation of high UV sensitive SiPMs from MEPhI/MPI for use in liquid argon
}

\section{Elena Popova ${ }^{1}$, Boris Dolgoshein ${ }^{\dagger}$, Pavel Buzhan, Andrey llyin, Alexey Stifutkin, Anatoly Pleshko}

National Research Nuclear University “MEPhI” 115409 Moscow Kashirskoe shosse 31, Russia

E-mail: elenap73@mail.ru

\section{Leonid Bezrukov, Bayarto Lubsandorzhiev, Nima Lubsandorzhiev}

Institute for Nuclear Research of RAS 117312 Moscow pr-t 60-letiya Oktyabrya 7a, Russia

E-mail: bezrukoveinr.ac.ru

\section{Razmik Mirzoyan}

Max-Planck-Institute for Physics 80805, Foehringer Ring 6, Munich, Germany

E-mail: Razmik.Mirzoyan@mpp.mpg.de

\section{Andrey Zhukov}

National Research University of Electronic Technology 124498 proezd 4806, building 5, Zelenograd, Russia

E-mail: a.zhukovetcen.ru

Abstract. The GERDA experiment requires large area photodetectors with sensitivity to $128 \mathrm{~nm}$ light and with extremely low radioactivity. Novel Silicon Photomultiplier (SiPM) is a promising candidate for this photodetector. Questions concerned with development of vacuum UV sensitive SiPMs with extremely large area are discussed with emphasis on electrical SiPM models and SPICE parameters extraction.

International Workshop on New Photon-detectors,

LAL Orsay, France

June 13-15, 2012

$1 \quad$ Speaker 


\section{Introduction}

Liquid Argon is used in the neutrinoless double beta-decay experiment GERDA (Germanium Detectors Array) [1]. The experimental setup consists of high purity Ge detectors array which is used simultaneously as a target and as a source of ${ }^{76} \mathrm{Ge}$ decay: ${ }^{76} \mathrm{Ge} \rightarrow{ }^{76} \mathrm{Se}+2 \mathrm{e}$. In this case we have a violation of lepton number conservation law, so if such decay exists it should be very rare. Therefore the experiment requires extremely low background conditions.

The germanium detectors operate in a cryostat filled with liquid Argon (LAr). High purity LAr is used as a cooling agent and as well as a passive shielding from external radioactivity.

At the time being the GERDA experiment is going to enter in phase II. One of the main goals of this phase is an instrumentation of LAr in order to suppress background to $\mathrm{B} \leq 10^{-}$ ${ }^{3} \mathrm{cts} /(\mathrm{kg} \cdot \mathrm{keV} \cdot \mathrm{y})$. It could be done by converting LAr from passive to active shielding by means of detection of LAr scintillation light caused by cosmic rays and internal radioactivity in LAr.

For LAr scintillation light registration there are 3 possible ways:

1) Wavelengths shifter fibers and cryogenic PMTs;

2) Wavelengths shifter fibers and cryogenic SiPMs;

3) Cryogenic extra UV SiPMs with large area.

Lets consider the last option. Extra UV SiPM should be sensitive to LAr light with wavelength $128 \mathrm{~nm}$ and has area more then $1 \mathrm{~cm}^{2}$.

For developing of such sensor we consider MEPhI/MPI SiPM as a prototype.

\section{UV sensitive SiPMs for GERDA}

MEPhI/MPI SiPMs have been produced in Zelenograd (Russia) in collaboration with Excelitas Techologies (former PerkinElmer) [2] as a result of long term cooperation between MEPhI and MPI for developing of SiPMs for Cherenkov telescopes applications [3,4]. Produced SiPMs have extended UV sensitivity suitable for Cherenkov light registration with Photon Detection Efficiency (PDE) of above $50 \%$ for light in a region $300-500 \mathrm{~nm}$. Simultaneously these SiPMs have a very low crosstalk at the level of several percents and extremely low sensitivity of gain to temperature variations, amounting to $0.5 \% /{ }^{\circ} \mathrm{C}$. SiPM's active area is up to $3 \times 3 \mathrm{~mm}^{2}[5]$.

However in order to use these devices for GERDA experiment we need to resolve at least 2 major problems.

The first one is to shift sensitivity to vacuum UV region, the second - to increase working area of SiPMs up to $1 \mathrm{~cm}^{2}$.

\subsection{Development of deep UV SiPMs}

SiPM's Photon Detection Efficiency (PDE) is directly determined by Quantum Efficiency QE. QE can be represented as: $Q E=T * I Q E$, where $T$ is transparency of SiPM's antireflective coating (ARC) and IQE is internal Quantum Efficiency. IQE is a probability for photogenerated 
carriers from undepleted parts of SiPM's entrance window to be extracted into high field region where Geiger discharge may arise due to impact ionization process.

Fig.1a shows calculated ARC transparency of actual MEPhI/MPI SIPMs. Calculations have been done by using specially written at MEPhI computer program. The program's extension to deep UV region is under development now.
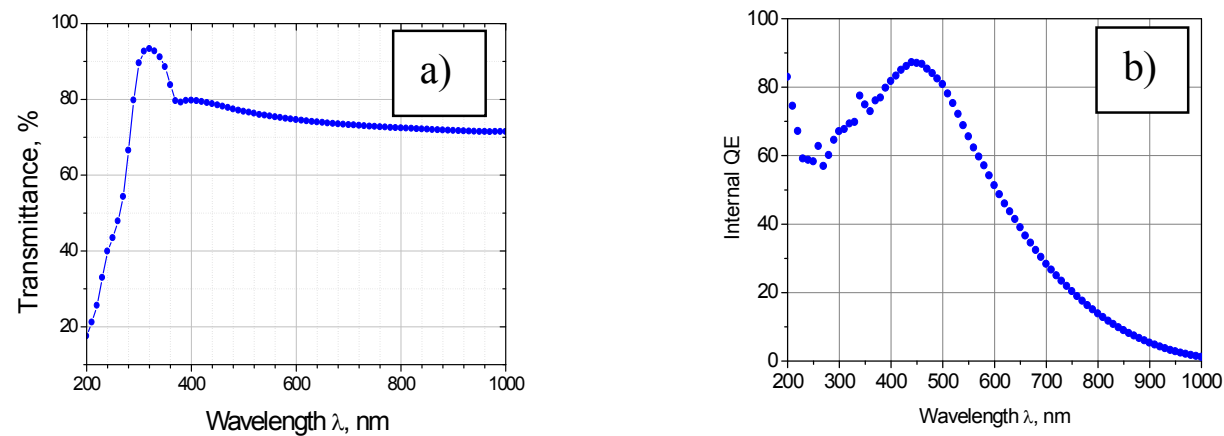

Fig.1. a) Calculated transmittance of ARC MEPhI/MPI SiPM.

b) Spectral dependence of Internal QE of MEPhI/MPI SiPM.

IQE of prototype devices is presented on fig.1b. One can see that devices already have quite high IQE at the level of $60-80 \%$ for $200 \mathrm{~nm}$ light. We are expecting that for $128 \mathrm{~nm}$ IQE will be even higher due to higher quantum yield for energetic UV light photons which can produce in silicon more then one electron-hole pair. It should be noted that the absorption length for deep UV light is almost constant for the region below $300 \mathrm{~nm}$ and roughly equal to $5-7 \mathrm{~nm}$ [6].

\subsection{Large area SiPMs}

The largest SiPM produced by MEPhI/MPI collaboration has sensitive area $5 \times 5 \mathrm{~mm}^{2}$ [4]. Already this size leads to a significant internal capacity of SiPMs which increase external preamplifier noise and impact a signal waveform. With SiPM sensitive area increase these problems will become more severe and require careful analysis and an electrical simulation of SiPM itself and corresponding FE electronics. To do this properly we have to develop an electrical SiPM model and establish precise procedure for SiPM SPICE parameters extraction.

\section{SiPM electrical model and parameters extraction}

Very simple SiPM electrical model has been proposed by F.Corsi and others several years ago [7]. For our SiPMs we have modified slightly that model by taking into account several new elements: pixel's spreading resistor $R_{s}$ and common inductance $L_{s}$ (aluminum buses inside SiPM, bonding wires and connection pins). Spreading resistor limits maximum value of the avalanche current inside high field region, inductance leads to oscillation process after Geiger discharge.

Our model is shown on fig. 2. $\mathrm{R}_{\mathrm{q} / \mathrm{n}}$ corresponds to quenching resistors of $\mathrm{n}$ fired pixels, $\mathrm{R}_{\mathrm{q} /(\mathrm{N}-\mathrm{n})}$ - resistors for non-fired pixels from $\mathrm{N}$ total number of pixels inside of SiPM. $\mathrm{C}_{\mathrm{q}^{*} \mathrm{n}}$ is a 
stray capacity between readout buses (metal lines) of SiPM and entrance window (upper electrode) of SiPM pixel, and $\mathrm{C}_{\mathrm{d}^{*} \mathrm{n}}$ is a capacity of p-n-junctions for $\mathrm{n}$ fired pixels. $\mathrm{C}_{\mathrm{q}^{*}(\mathrm{~N}-\mathrm{n})}$ and $\mathrm{C}_{\mathrm{d}^{*}(\mathrm{~N}-\mathrm{n})}$ are the same parameters for the remaining (non fired) pixels in SiPM. $\mathrm{C}_{\mathrm{g}}$ is a total capacity of metal buses and connection pins.

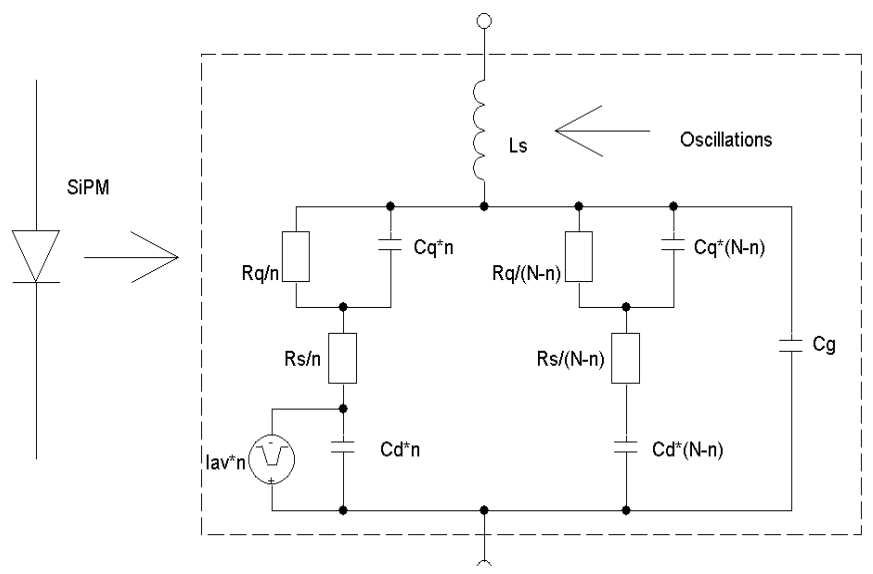

Fig.2. MEPhI/MPI SiPM equivalent scheme.

For extraction of parameters we consider 3 different ways: SiPM waveform analysis, high intensity double light pulses and extraction by using network analyzer. It should be noted that the most difficult parameter to extract is $\mathrm{C}_{\mathrm{q}}$. Even for SiPM designers who exactly know the topology of the device and production technology used estimation of $\mathrm{C}_{\mathrm{q}}$ value is rather complicated task.

\subsection{SiPM waveform analysis}

Waveform analysis is the simplest technique used for SiPM electrical parameters extraction, it requires only oscilloscope and fast light source (sub-ns time region) without "tail" in light pulses. Also diffusion processes for carriers from undepleted regions inside SiPM should be taken into account. Then care must be taken to avoid external circuit influence on analyzed waveform (high bandwidth of preamplifier and low value load resistor $\mathrm{R}_{\text {load }}$ which is usually $50 \mathrm{Ohm}$ in order to provide $\mathrm{R}_{\mathrm{q}} / \mathrm{N}>>\mathrm{R}_{\text {load }}$ conditions are required). It means that before starting of experiments $\mathrm{R}_{\mathrm{q}}$ should be estimated by direct voltage-current measurements.

Waveform of SiPM signal consists of 2 parts: fast one is caused by Geiger discharge itself and slow one corresponds to pixel potential restoration after Geiger discharge. "Slow" part characteristic time $\tau_{\mathrm{r}}$ depends on total pixel capacity $\mathrm{C}_{\text {tot }}=\mathrm{C}_{\mathrm{q}}+\mathrm{C}_{\mathrm{d}}$ and resistivity of pixel quenching resistor $\mathrm{R}_{\mathrm{q}}$ :

$$
\tau_{\mathrm{r}}=\mathrm{R}_{\mathrm{q}}\left(\mathrm{C}_{\mathrm{q}}+\mathrm{C}_{\mathrm{d}}\right) \text {. }
$$

An example of waveform for $1 \mathrm{x} 1 \mathrm{~mm}^{2} \mathrm{UV}$ SiPM with $100 \mathrm{x} 100$ micron pixel size manufactured in Zelenograd (type 100B) is presented on insertion of fig.3a. One can see the "fast" and "slow" parts on this scope signal. Charge relations connected with such 2 parts are:

$\mathrm{Q}_{\text {tot }}=\Delta \mathrm{U}^{*}\left(\mathrm{C}_{\mathrm{q}}+\mathrm{C}_{\mathrm{d}}\right) ; \mathrm{Q}_{\text {fast }}=\Delta \mathrm{U}^{*} \mathrm{C}_{\mathrm{q}} ; \mathrm{Q}_{\text {slow }}=\Delta \mathrm{U}^{*} \mathrm{C}_{\mathrm{d}}$, where $\mathrm{Q}_{\text {tot }}=\mathrm{Q}_{\text {fast }}+\mathrm{Q}_{\text {slow }}$ - total charge produced during Geiger discharge for overvoltage $\Delta \mathrm{U}, \mathrm{Q}_{\text {fast }}$ and $\mathrm{Q}_{\text {slow }}$ charge in "fast" and "slow" components correspondingly. 
It should be noted that for some cases separation between "fast" and "slow" part is rather poor.

For our device "slow" part of the pixel signal has small amplitude $A=\Delta U / R_{q}$ due to relative high value of a quenching resistor and in order to make it more visible at the noise level we have used a fast blue $(405 \mathrm{~nm})$ laser with light signal duration of about $40 \mathrm{ps}$. Intensity of light pulses was attenuated by using neutral filters so that number of fired pixels changed from $1 / 4$ of total number of SiPM pixels to full saturation (fig.3b). Slow part of signal had been fitted by exponential function.
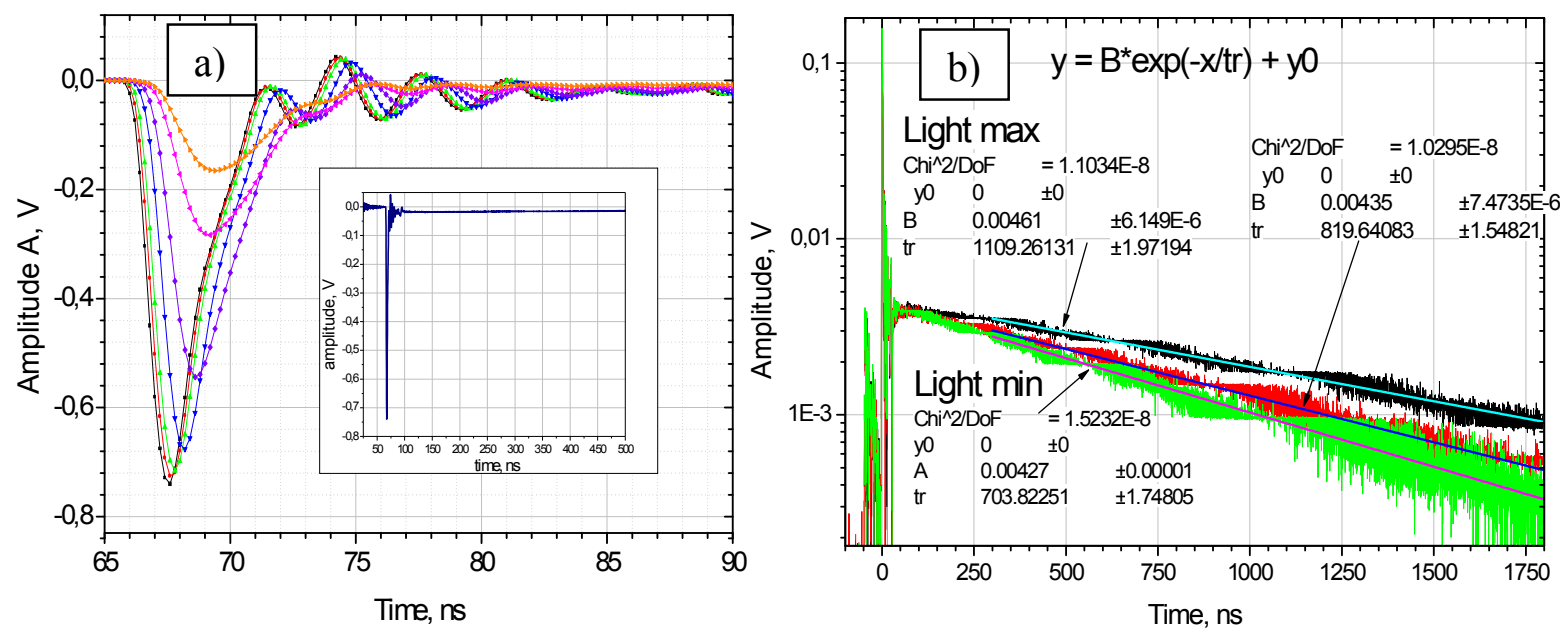

Fig.3. a) MEPhI/MPI SiPM signal for different light pulse intensities.

b) "Slow" part of MEPhI/MPI SiPM signal for different light pulse intensities

We observed that restoration time $\tau_{\mathrm{r}}$ defined in (1) is sensitive to light intensity when SiPM became saturated. On fig.4a one can see a dependence of $\tau_{\mathrm{r}}$ from amplitude of SiPM pulse. So we propose to use restoration time which doesn't depend from light intensity (at the plateau region) and is equal $720 \pm 40 \mathrm{~ns}$. Increasing of $\tau_{\mathrm{r}}$ with light intensity could possibly be explained by modulation of total pixel capacity by free carriers produced due to photoeffect and impact ionization.
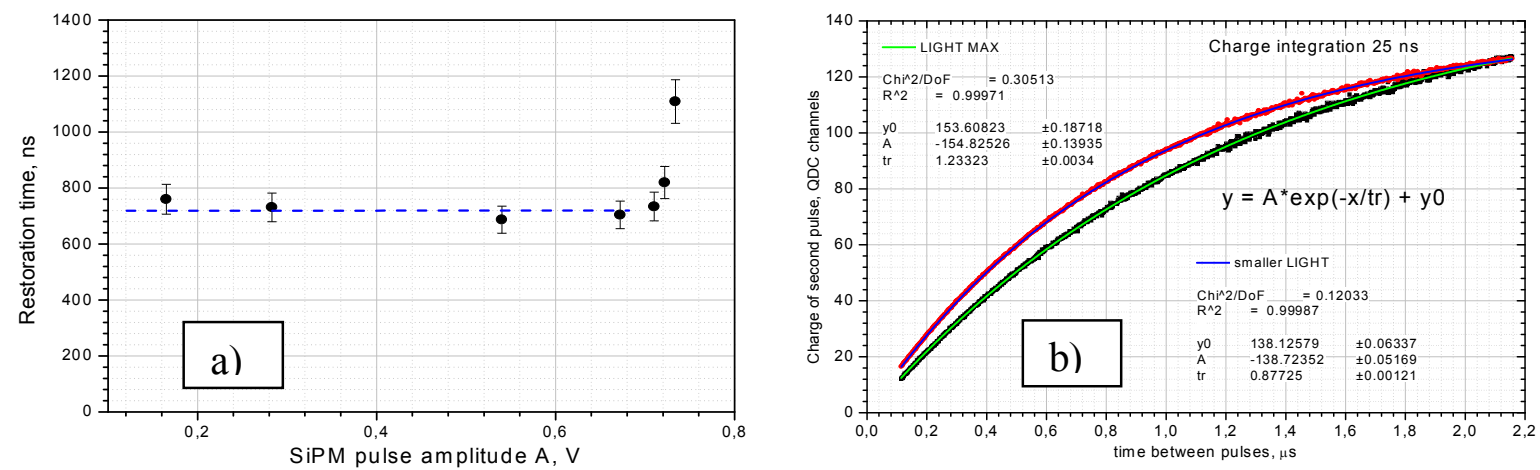

Fig.4. a) Dependence of $\tau_{\mathrm{r}}$ from amplitude of SiPM pulse caused by laser light of different intensities.

b) Second pulse amplitude vs delay between double light pulses for different light intensities. 


\subsection{High intensity double light pulses technique}

For such technique one needs to use 2 consequent light pulses with high intensity in order to fire all pixels inside SiPM. The amplitude of a SiPM second pulse is measured versus time interval between the pulses. Such method doesn't demand clear visibility of SiPM waveform but to obtain the correct amplitude of second pulse tail of SiPM signal caused by first light pulse must be taken into account.

Restoration time $\tau_{\mathrm{r}}$ is a result of fitting procedure of the second pulse amplitude vs time. Fitting function is first order exponential decay. We observed that $\tau_{\mathrm{r}}$ for our sample depends from light intensity too and in a good accordance with previous measurements based on waveform analysis (fig.4b).

\subsection{Network analyzer measurements}

The network analyzer 4287A AGILENT provides measurement of complex impedance $Z_{e}\left(f_{n}\right)$ for number of different frequencies $\left(f_{n}\right)$ which have an advantage from the point of view of precision when compared with one frequency measurements [7]. We assume SiPM connection and equivalent SiPM electrical scheme for impedance measurement are as shown on fig. 5a, so

$Z\left(f_{n}\right)=R\left(f_{n}\right)+j X \quad\left(f_{n}\right)$,

where $\mathrm{R}$ and $\mathrm{X}$ are real and imaginary parts of complex impedance correspondingly. On the basis of equivalent scheme we can derive:

$R\left(\omega^{2}\right)=R_{d} \frac{C_{d}^{2}}{\left(C_{d}+C_{3}\right)^{2}+\omega^{2}\left(C_{3} R_{d} C_{d}\right)^{2}}, \quad X(\omega)=\frac{1-\omega L C^{\prime}}{\omega C^{\prime}}$,

where $\omega=2 \pi \mathrm{f}$,

$C^{\prime}=\frac{\left(C_{d}+C_{3}\right)^{2}+\omega^{2}\left(C_{3} R_{d} C_{d}\right)^{2}}{C_{d}+C_{3}+\omega^{2} C_{3}\left(R_{d} C_{d}\right)^{2}}, \quad C_{d}\left(\omega^{2}\right)=C_{2} \frac{1+\omega^{2}\left(R_{1} C_{1}\right)^{2}}{1+\omega^{2}\left(R_{1}\right)^{2} C_{1}\left(C_{1}+C_{2}\right)}, R_{d}\left(\omega^{2}\right)=\frac{R_{1}}{1+\omega^{2}\left(R_{1} C_{1}\right)^{2}}+R_{2}$,

here $\mathrm{R}_{1}=\mathrm{R}_{\mathrm{q}} / \mathrm{N}, \mathrm{C}_{1}=\mathrm{C}_{\mathrm{q}} \times \mathrm{N}, \mathrm{R}_{2}=\mathrm{R}_{\mathrm{s}} / \mathrm{N}, \mathrm{C}_{2}=\mathrm{C}_{\mathrm{d}} \times \mathrm{N}$, parasitic elements are $\mathrm{C}_{3}$ and $\mathrm{L}$ (pins for connection SiPM to network analyzer).

To extract SiPM electrical parameters we have performed minimization of function

$$
Q\left(\omega_{1}, \ldots \omega_{N}, R_{1}, R_{2}, C_{1}, C_{2}, C_{3}, L\right)=\sum_{n=1}^{15}\left\{\frac{\left[R_{T}\left(\omega_{n}^{2}\right)-R_{E}\left(\omega_{n}^{2}\right)\right]^{2}+\left[X_{T}\left(\omega_{n}\right)-X_{E}\left(\omega_{n}\right)\right]^{2}}{R_{E}^{2}\left(\omega_{n}^{2}\right)+X_{E}^{2}\left(\omega_{n}\right)}\right\}
$$

for all 15 frequencies, where $\mathrm{E}_{\mathrm{E}}$ indexes correspond to experimental data and $\mathrm{T}^{-}$to searched for values.

Low frequencies data depend on capacity of pixel's p-n-junction $\left(\mathrm{C}_{\mathrm{d}}\right)$, high frequencies data are significantly affected by stray capacity $\left(\mathrm{C}_{\mathrm{q}}\right)$.

In order to improve accuracy of extracted parameters during the fitting procedure we have fixed quenching resistors value $\mathrm{R}_{1}=7,85 \mathrm{kOhm}$ (derived from voltage-current measurements for direct bias) and p-n-junction capacity $\mathrm{C}_{2}=75 \mathrm{pF}$ (obtained for reverse bias close to breakdown voltage for $25 \mathrm{~Hz}$ ) for whole SiPM. Fig. $5 \mathrm{~b}$ shows the fitting result. Now we can extract SPICE parameters for an individual pixel: $\mathrm{C}_{\mathrm{q}}=\mathrm{C}_{1} / 100=(190 \pm 40) \mathrm{fF}, \mathrm{C}_{\mathrm{d}}=\mathrm{C}_{2} / 100=(750 \pm 10) \mathrm{fF}$, $\mathrm{R}_{\mathrm{q}}=\mathrm{R}_{1} * 100=785 \mathrm{kOhm}, \mathrm{R}_{\mathrm{s}}=\mathrm{R}_{2} * 100=2.2 \mathrm{kOhm}, \mathrm{C}_{\mathrm{g}}=\mathrm{C}_{3} \approx 0, \mathrm{~L}_{\mathrm{s}}=\mathrm{L}=(6 \pm 1) \mathrm{nH}$. It gives us the value for $\tau_{\mathrm{r}}=(740 \pm 40) \mathrm{ns}$ which is in a good agreement with value obtained from waveform analysis. 

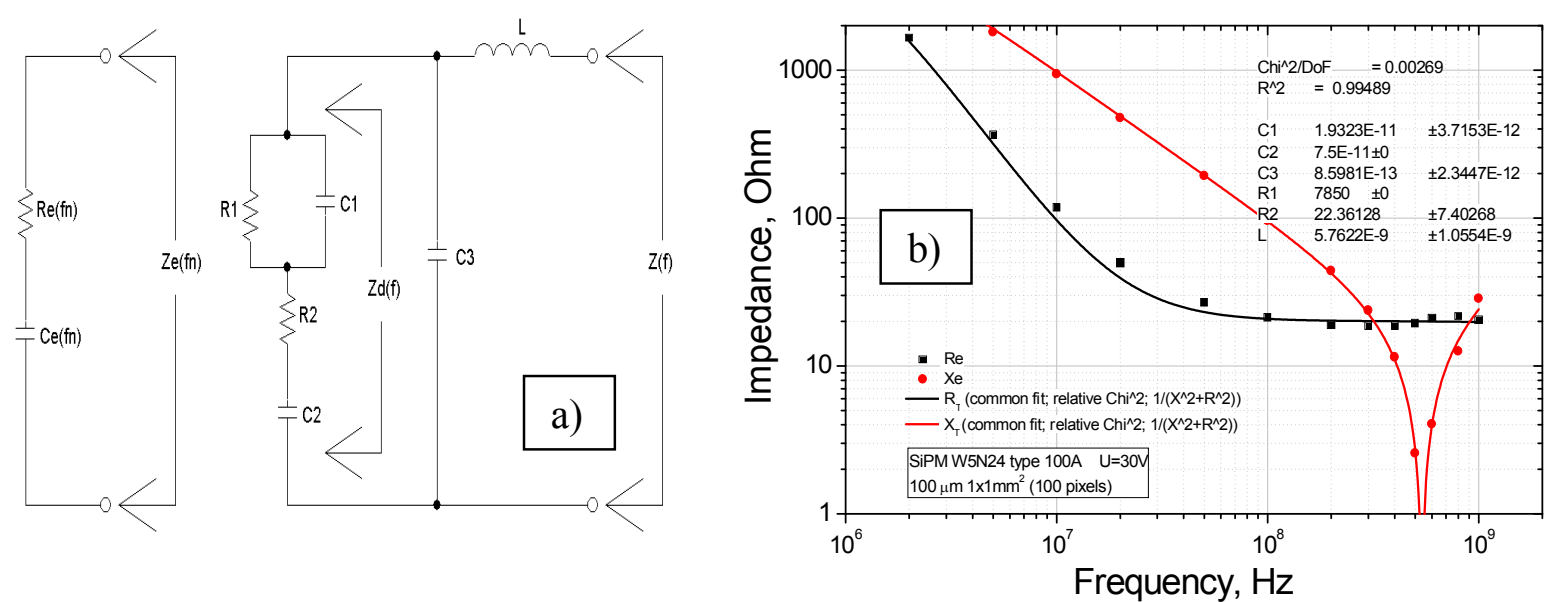

Fig.5. a) Connection diagram and electrical equivalent scheme for complex impedance measurements with network analyzer.

b) Fit result of complex impedance measurements of MEPhI/MPI SiPM.

\section{Conclusions}

The development of vacuum UV sensitive SiPMs for direct LAr scintillation light registration in the GERDA experiment has good perspectives.

Very frequently used methods for SiPM SPICE parameters extraction based on waveform analysis and double pulses technique require careful analysis for validity of application. They have certain limitations and could in addition be sensitive to light intensity. New experimental technique based on the complex impedance measurements for a number of frequencies is proposed and proved experimentally.

\section{References}

[1] I.Abt et al., GERDA Collaboration, GERDA: the Germanium detector array, proposal http://www.mpi-hd.mpg.de/GERDA

[2] www.excelitas.com

[3] B.Dolgoshein et al., SiPM and ADD as advanced detectors for astro-particle physics, Nucl. Phys.A 572 (2007) 493-494

[4] B.Dolgoshein et al., The cross-talk problem in SiPMs and their use as light sensors for imaging atmospheric Cherenkov telescopes, Nucl. Phys. A 610 (2009) 131-134

[5] B.Dolgoshein et al., Large area UV SiPMs with extremely low cross-talk, Nucl. Phys. A695 (2012) 40-43

[6] D. Edwards, Silicon(Si), in Handbook of Optical Constants of Solids V1 edited by Edward D. Palik Academic Press, USA 1998

[7] F. Corsi et al., Modelling a silicon photomultiplier ( $\mathrm{SiPM})$ as a signal source for optimum frontend design, Nucl. Phys. A 572 Issue 1 (2007) 416-418 